\title{
How does the visual cortex of the blind acquire auditory responsiveness?
}

\begin{abstract}
Michael Wong ${ }^{*}$ and Arindam Bhattacharje ${ }^{2}$
1 McMaster Integrative Neuroscience Discovery and Study, McMaster University, Hamilton, ON, Canada

2 Department of Psychology, Neuroscience and Behaviour, McMaster University, Hamilton, ON, Canada

*Correspondence:wongm8@mcmaster.ca
\end{abstract}

\section{A commentary on}

Corticocortical connections mediate primary visual cortex responses to auditory stimulation in the blind

by Klinge, C., Eippert, F., Röder, B., and Büchel, C. (2010). J. Neurosci. 30, 12798-12805.

In blindness, the primary visual cortex (V1) responds to auditory inputs (cross-modal plasticity; Kupers et al., 2011), but where do these auditory inputs originate? In a study published in the Journal of Neuroscience, Klinge et al. (2010) performed dynamic causal modeling (DCM) on functional magnetic resonance imaging (fMRI) data to investigate the origin of auditory inputs driving $\mathrm{V} 1$ activation in blind human participants. The authors concluded, based on their modeling results, that $\mathrm{V} 1$ activation in the blind is most likely driven by auditory inputs arising from the primary auditory cortex (A1).

Although Klinge et al. (2010) suggest a functional link between $\mathrm{A} 1$ and V1 in the blind, it is difficult to know based on fMRI and modeling data alone how these two areas might be anatomically connected. Interestingly, the effective connectivity between sensory areas in blind adult humans, as concluded by Klinge et al. (2010), is similar to the anatomical connectivity between sensory areas in blind adult (non-primate) animals, as reported in neuroanatomical tracing studies. While we acknowledge the caveat of generalizing neuroanatomical tracing data obtained from non-primate species to humans, we believe that these tracing data when complemented with the data of Klinge et al. (2010) can provide important insights into the anatomical connectivity between sensory areas in blind humans. Our aim in this commentary is therefore to discuss the conclusions of Klinge et al. (2010) in light of the published animal literature, focusing specifically on congenital and early blindness.
Klinge et al. (2010) used fMRI to measure the blood oxygen-level dependent activity of congenitally blind and normally sighted human participants during an auditory non sense-word-discrimination task, and then used DCM (see Friston, 2009) to infer, from the fMRI data, the effective connectivity between three hypothesized brain regions: $\mathrm{V} 1, \mathrm{~A} 1$, and the medial geniculate nucleus (MGN). The authors considered eight models, each differentially explaining the effective connectivity among those three hypothesized brain regions. These models ranged from a simple feedforwardonly model, with inputs from the MGN to both A1 and V1, to a complicated fully connected model, with both feedforward and backward inputs among the three regions (see Klinge et al., 2010, Figure 1). The authors first established the most probable model within each hemisphere in each vision group by comparing the eight models to one another based on two criteria: (1) each model's complexity, and (2) how well each model explains the data. The authors found that the fully connected model was the most probable model for both hemispheres in both vision groups. This suggests that there exist reciprocal connections among the three hypothesized brain regions in both blind and sighted participants.

Klinge et al. (2010) found further that the $\mathrm{A} 1-\mathrm{V} 1$ connection strength was greater in blind than in sighted participants. The results of tracing studies have revealed connections from A1 to V1 in sighted animals (macaque monkeys: Falchier et al., 2002; opossums: Kahn et al., 2000; Karlen et al., 2006; cats: Innocenti et al., 1988). Interestingly, Karlen et al. (2006) and Innocenti et al. (1988) observed that these connections were more abundant in neonatally enucleated than in sighted opossums and cats. Thus, the greater A1-V1 connection strength in blind than in sighted participants, reported by Klinge et al. (2010), possibly reflects the presence of more A1V1 connections in blind than in sighted human participants.

The authors then found, although nonsignificant, a trend for stronger MGN-V1 connection strength in blind than in sighted participants in the right hemisphere. This non-significant trend is particularly interesting in light of tracing studies that have reported the existence of thalamocortical connections from the MGN to V1 in mutant congenitally eyeless mice (Laemle et al., 2006) and - although scarce - in neonatally enucleated opossums (Karlen et al., 2006). Perhaps the non-significant trend reported by Klinge et al. (2010) reflects the existence of, albeit scarce, connections between the MGN and V1 in blind human participants.

Thus far, the conclusions of Klinge et al. (2010) agree well with the results of tracing studies, suggesting that there exist corticocortical and possibly thalamocortical connections from $\mathrm{A} 1$ and $\mathrm{MGN}$ respectively to V1 in the blind. Next, Klinge et al. (2010) questioned whether the LGN might relay auditory inputs to V1 in the blind, perhaps via the inferior colliculus (IC). The authors found no difference in the LGN-V1 connection strength between blind and sighted participants. This suggests that auditory inputs from the LGN do not drive V1 activation in blind humans, presumably reflecting the absence of IC-LGN connections. Agreeing closely with this conclusion, Chabot et al. (2007) did not observe anatomical connections from the IC to LGN in neonatally enucleated mice. Note, however, that these peculiar connections have been observed in mutant congenitally eyeless mice (Chabot et al., 2007, 2008) and neonatally enucleated hamsters (Izraeli et al., 2002).

A hypothesis that was not considered by Klinge et al. (2010) is that V1 activation in the blind might be driven by auditory inputs arising exclusively, or partly, from multimodal areas of the cortex (e.g., auditory association areas). Tracing studies have 
reported connections from multimodal areas to $\mathrm{V} 1$ in sighted macaque monkeys (Rockland and Ojima, 2003) and opossums (Karlen et al., 2006). Karlen et al. (2006) further observed that such connections were more abundant in neonatally enucleated than sighted opossums. Because Klinge et al. (2010) did not include multimodal areas in any of their eight models, these areas cannot be ruled out as potential sources of auditory inputs driving V1 activation in blind participants.

An interesting avenue for future research would be to investigate whether the connections reported by Klinge et al. (2010) are dependent on the age of blindness onset, as the authors only investigated a sample of congenitally blind participants. A developmental study on cats has shown that although there are connections from the auditory cortex to visual cortex early in life, many of these connections are pruned during development, presumably by visual experience (Innocenti et al., 1988). This suggests that the connectivity between sensory areas in humans who become blind as adults might more closely resemble that of sighted than congenitally blind participants. If connections between V1 and auditory areas are scarce in the late blind, how does V1 acquire sufficient auditory inputs to drive cross-modal plasticity? One possibility is that in the absence of competing visual inputs the scarce connections between V1 and auditory areas are unmasked and/or strengthened. Alternatively, blindness may induce the formation of new connections that link auditory areas to V1 (see Kupers et al., 2011). Future studies should investigate these possibilities.

Although it has been previously hypothesized that $\mathrm{V} 1$ activation in the blind might be driven by auditory inputs from A1 (Bavelier and Neville, 2002), Klinge et al. (2010) were the first to systematically investigate and provide evidence supporting this hypothesis in blind humans. We note further that the conclusions drawn by Klinge et al. (2010) from their modeling results and the findings reported in tracing studies are similar, suggesting that blindness induces similar structural reorganization in most mammalian species, and that the physiological mechanism underlying this form of reorganization might be general.

\section{ACKNOWLEDGMENTS}

We thank Daniel Goldreich, Jonathan Tong, Ryan Peters, Vani Pariyadath, and Kathleen S. Rockland for their many helpful comments.

\section{REFERENCES}

Bavelier, D., and Neville, H. J. (2002). Cross-modal plasticity: where and how? Nat. Rev. Neurosci. 3, 443-452.

Chabot, N., Charbonneau, V., Laramee, M. E., Tremblay, R., Boire, D., and Bronchti, G. (2008). Subcortical auditory input to the primary visual cortex in anophthalmic mice. Neurosci. Lett. 433, 129-134.

Chabot, N., Robert, S., Tremblay, R., Miceli,D., Boire, D., and Bronchti, G. (2007).Audition differently activates the visual system in neonatally enucleated mice compared with anophthalmic mutants. Eur. J. Neurosci. 26, 2334-2348.

Falchier, A., Clavagnier, S., Barone, P., and Kennedy, H. (2002). Anatomical evidence of multimodal integration in primate striate cortex. J. Neurosci. 22, 5749-5759.
Friston, K. (2009). Causal modelling and brain connectivity in functional magnetic resonance imaging. PLoS Biol. 7, e33. doi:10.1371/journal.pbio.1000033

Innocenti, G. M., Berbel, P., and Clarke, S. (1988). Development of projections from auditory to visual areas in the cat. J. Comp. Neurol. 272, 242-259.

Izraeli, R., Koay, G., Lamish, M., Heicklen-Klein, A. J., Heffner, H. E., Heffner, R. S., and Wollberg, Z. (2002). Cross-modal neuroplasticity in neonatally enucleated hamsters: structure, electrophysiology and behaviour. Eur. J. Neurosci. 15, 693-712.

Kahn, D. M., Huffman, K. J., and Krubitzer, L. (2000). Organization and connections of V1 in Monodelphis domestica. J. Comp. Neurol. 428, 337-354.

Karlen, S. J., Kahn, D. M., and Krubitzer, L. (2006). Early blindness results in abnormal corticocortical and thalamocortical connections. Neuroscience 142, 843-858.

Klinge, C., Eippert, F., Roder, B., and Buchel, C. (2010). Corticocortical connections mediate primary visual cortex responses to auditory stimulation in the blind. J. Neurosci. 30, 12798-12805.

Kupers, R., Pietrini, P., Ricciardi, E., and Ptito, M. (2011). The nature of consciousness in the visually deprived brain. Front. Psychol. 2:19. doi: 10.3389/ fpsyg.2011.00019

Laemle, L. K., Strominger, N. L., and Carpenter, D. O. (2006). Cross-modal innervation of primary visual cortex by auditory fibers in congenitally anophthalmic mice. Neurosci. Lett. 396, 108-112.

Rockland, K. S., and Ojima, H. (2003). Multisensory convergence in calcarine visual areas in macaque monkey. Int. J. Psychophysiol. 50, 19-26.

Received: 23 June 2011; accepted: 27 July 2011; published online: 11 August 2011.

Citation: Wong Mand Bhattacharjee A (2011) How does the visual cortex of the blind acquire auditory responsiveness? Front. Neuroanat. 5:52. doi: 10.3389/fnana.2011.00052 Copyright $(\odot 2011$ Wong and Bhattacharjee. This is an openaccess article subject to a non-exclusive license between the authors and Frontiers Media SA, which permits use, distribution and reproduction in other forums, provided the original authors and source are credited and other Frontiers conditions are complied with. 